Published in final edited form as:

Hepatology. 1990 February ; 11(2): 159-164.

\title{
Orthotopic Liver Transplantation for Alcoholic Liver Disease
}

\author{
Shashi Kumar, Rudolf E. Stauber, Judith S. Gavaler, Michael H. Basista, Vincents J. \\ Dindzans, Robert R. Schade, Mordechai Rabinovitz, Ralph E. Tarter, Robert Gordon, Thomas \\ E. Starzl, and David H. Van Thiel \\ Departments of Epidemiology, Medicine, Psychiatry, and Surgery, University of Pittsburgh, School \\ of Medicine, Pittsburgh, PA 15261.
}

\section{Abstract}

\begin{abstract}
Alcohol abuse is the most common cause of end-stage liver disease in the United States, but many transplant centers are unwilling to accept alcoholic patients because of their supposed potential for recidivism, poor compliance with the required immunosuppression regimen and resulting failure of the allograft. There is also concern that alcohol-induced injury in other organs will preclude a good result. From July 1, 1982, to April 30, 1988, 73 patients received orthotopic liver transplants at the University of Pittsburgh for end-stage alcoholic liver disease. Fifty-two (71\%) of these were alive at $25 \pm 9$ mo (mean \pm S.D.) after transplantation, when a phone survey of these patients, their wives/ husbands, and their physicians was performed to evaluate their subsequent use of alcohol, current medical condition and employment. Data obtained were compared with those for nonalcoholic patients selected as transplant controls. The recidivism rate has been $11.5 \%$, with most patients drinking only socially. Fifty-four percent of the survivors are employed, 21\% classify themselves as homemakers and only $11(21 \%)$ are unable to work. Twenty-one patients died after transplantation; the most frequent cause of death was sepsis (43\%), and intraoperative death was the next most common cause $(28.6 \%)$. These data demonstrate that alcoholic patients can be transplanted successfully and achieve good health not significantly different from that of individuals transplanted for other causes. Thus orthotopic liver transplantation is a therapeutic option that should be considered for individuals with end-stage alcoholic liver disease who desire such therapy.
\end{abstract}

Orthotopic liver transplantation (OLT) is a therapeutic option for persons with end-stage liver disease that no longer responds to medical treatment (1-3). Alcohol-induced cirrhosis and alcoholic hepatitis are two of the most common forms of fatal liver disease seen in the United States (4). Both conditions can be treated with orthotopic liver transplantation. Because alcohol-induced liver injury is the most frequent cause of cirrhosis in the United States and other western countries, most candidates for OLT are alcoholics (5). Few centers, however, have been willing to provide transplants to persons with alcohol-induced liver disease. The major reason for this reluctance has been the fear of a high rate of recidivism that might lead to disease in the allograft. This opinion persists even though little or no data exist concerning the recurrence of alcohol abuse after liver transplantation. This study was undertaken to provide some data relative to these issues.

\section{MATERIALS AND METHODS}

From July 1, 1982, to April 30, 1988, 73 persons with advanced alcoholic liver disease underwent OLT at the hospital of the University of Pittsburgh's School of Medicine, for end-

Copyright (c) 1990 the American Association for the Study of Liver Diseases

Address reprint requests to: D.H. Van Thiel, M.D., 1000 J Scaife Hall, University of Pittsburgh, School of Medicine, Pittsburgh, PA $15261 .$. 
stage alcoholic liver disease. This is not our entire experience to date with alcoholic liver disease; it does, however, include an adequate number of patients and duration of time after transplant to predict long-term outcome and to make valid inferences about the larger entire population (Fig. 1). The diagnosis of alcoholic liver disease in each case was based on a history of habitual and excessive alcohol consumption in the absence of other causes of liver disease, compatible clinical and laboratory findings, pretransplant liver biopsy results and the results of the examination of the resected diseased liver after transplantation.

Survivors, their spouses and their local physicians were contacted by a protocol phone survey consisting of questions regarding current alcohol and illicit drug consumption, health status, compliance with the immunosuppression regimen and employment status since

transplantation. For the purposes of this study, social drinking was defined as the consumption of alcohol only on holidays and special occasions, moderate drinking was defined as three or fewer drinks per week and drinking above this level was defined as excessive. The subject's most recent serum transaminase levels were obtained from the clinic at the University of Pittsburgh or from his/her primary care physician. The control group consisted of an equal number of age-matched and sex-matched control patients transplanted during the same period by the same team of physicians and surgeons for end-stage hepatocellular liver disease not resulting from alcohol consumption.

\section{Statistical Analysis}

Patient survival was calculated by life table analysis using the Kaplan-Meier method; the survival curves for the two groups of patients were compared by the log-rank test. The chisquare test with Yates' adjustment was used to test differences between proportions and the significance of associations. A probability value of $\mathrm{p}<0.05$ was considered to be significant.

\section{RESULTS}

\section{Pretransplant Characteristics}

The mean age of the alcoholic transplant recipient group at the time of OLT was $48.16 \pm 8.69$ $\mathrm{yr}(\mathrm{mean} \pm$ S.D.), with a range of 21 to $70 \mathrm{yr}$. Men (79\%) predominated in the sample (Fig. 2). Sixty-four percent of the alcoholic patients transplanted were classified as being in Child's class C, $32 \%$ were class B and $4 \%$ were class A before transplantation (Table 1). An exact determination of the period of abstinence from alcohol before transplantation was impossible to determine, but most had ceased their alcohol consumption at least 6 mo before transplantation (Table 2). Only 7 of the 52 (13\%) survivors and 4 (19\%) in the deceased group admitted to having consumed alcohol in the 6 mo before liver transplantation. This difference was not statistically significant.

\section{Survival Analysis}

Fifty-two individuals (71\%) were alive in April 1989 after OLT for alcoholic liver disease. Survival at $1 \mathrm{yr}$ (Fig. 3), as calculated by the actuarial survival method, was $74 \%$, a figure similar to that for the age- and sex-matched control patients receiving transplants for nonalcohol-induced hepatocellular liver diseases at this institution. Median survival time at the time of this survey was $18 \mathrm{mo}$ for the alcoholic patients and $16 \mathrm{mo}$ for the nonalcoholic patients, a difference that was not significant.

\section{Causes of Death}

Of the 21 individuals who died as a result of liver transplantation, $6(29 \%)$ died intraoperatively, $7(33 \%)$ died in the immediate postoperative period and $8(38 \%)$ died 3 or more mo after transplantation. Four of these died of sepsis and only two died of rejection (Table 3). Thus the 
most frequent cause of death was sepsis ( 9 of 21), with intraoperative deaths ( 6 of 21) being second, followed by rejection ( 2 of 21) and a variety of other causes ( 4 of 21) (Table 3). Most of the individuals $(73 \%)$ who died after OLT were Child's class C.

\section{Recidivism}

Of the 52 surviving recipients, only 6 (11.5\%) have resumed alcohol consumption (Table 4). Four drink socially, and a fifth, who began drinking 4 mo after transplantation is consuming alcohol in moderate amounts (three or fewer drinks per week). This individual, at the time of this survey, was selling lottery tickets in a bar. The sixth individual has recently reduced his alcohol intake from moderate to social amounts after his physician warned him of test results indicating possible alcohol-induced liver injury.

Only two discrepancies were noted, and in each case the physician felt that the recipient was drinking but both patient and spouse denied any alcohol consumption was taking place (Table 5). The rate of recidivism was $43 \%$ in individuals who stopped drinking for less than 6 mo before OLT as compared with $6.7 \%$ for individuals who stopped drinking for more than 6 months; this difference is statistically significant $(\mathrm{p}<0.05)$. Of the four who died after discharge from the hospital one had resumed alcohol consumption and eventually died of allograft rejection; the rejection was at least in part a result of his noncompliance with immunosuppression and his recurrent alcohol abuse.

\section{Employment Status}

Before their transplants, the 73 patients had been unemployed for periods ranging from several months to several years. Thirteen of the 52 survivors $(25 \%)$ at time of follow-up were currently working full-time, 10 were working part-time (19\%) and 5 were self-employed (9.6\%). Eleven were homemakers $(21 \%)$, and 11 were unable to work (21\%). Nine of the 11 who claimed to be homemakers are women. The remaining two patients are willing to work but have not been able to find employment (Table 6). Twenty-two of these 28 employed patients required at least 6 mo of recuperation after their transplant operation before they could return to work. The percentage of working transplant survivors is no different from that seen in patients transplanted for diseases other than alcoholism.

\section{Marital Status}

Forty-five of the 52 surviving patients (86.5\%) are married, 3 are divorced (5.7\%), 3 are single $(5.7 \%)$ and 1 is widowed (1.9\%) (Table 7). It is notable that the alcoholic population had a trend toward a higher percentage of married patients. There was no significant change in the marital status after OLT.

\section{Current Liver Enzyme Levels}

The most recent aminotransferase levels (AST and ALT) obtained for each of the 52 surviving recipients transplanted for alcoholic liver disease are shown in Table 8. At the time of the survey, 36 transplanted patients had normal enzymes and 16 individuals had minor elevations of their serum aminotransferase levels. Only one individual had an AST/ALT ratio greater than 2 , suggesting alcoholic liver disease. This patient was known to and admitted to be drinking alcohol in moderate amounts.

\section{DISCUSSION}

In June 1983 the National Institutes of Health convened a consensus development panel to develop guidelines for liver transplantation (6). In the section related to alcoholic liver disease, the panel attributed at least $50 \%$ of the cases of cirrhosis in the United States to alcohol abuse 
and named alcoholism as the leading cause of hepatic morbidity and mortality in this country. Importantly, they did not state that alcoholics should be excluded from liver transplantation. In fact, they suggested quite the opposite: that liver transplantation might be considered for patients in whom evidence of progressive liver failure has developed despite available current medical treatment and abstinence from alcohol. Relatively few patients with alcoholic liver disease have been transplanted, and an increase in the number of chronic alcoholics being transplanted has not been seen, despite the increase in the number of transplant centers (5).

It is generally held that clinically advanced alcoholic liver disease carries a 5-yr survival rate of only $50 \%$ (7), a prognosis worsened by the presence of ascites, portal hypertension, bleeding varices, hepatic encephalopathy and hepatorenal syndrome. Before the development of OLT, no specific therapy for alcoholic liver disease other than the cessation of alcohol intake existed. This still remains the "ideal" therapy, but even alcohol abstinence may not allow survival in patients with far advanced disease. It has been shown that once a certain level of hepatic damage has been reached, abstinence has no impact on survival $(8,9)$. In such cases, liver transplantation is the only option for long-term survival.

The major argument against the widespread application of liver transplantation for alcoholic patients has been a possible high rate of recidivism that would prevent long-term survival and lead to redevelopment of the original liver disease in the allograft. Another argument has been that alcoholic patients, because they often have accompanying multiorgan disease, have only a poor chance for a successful outcome and a return to employment. Thus the major factors determining the candidacy of patients with alcohol-induced liver disease for OLT have been related to aspects other than medical issues.

Most liver transplantation centers have set an arbitrary period of abstinence, usually as a requirement before an alcoholic patient can be a candidate for transplantation. Although abstinence is clearly of value in arresting alcoholic liver disease that is not end-stage, the benefit of alcohol abstinence before transplantation is undocumented. Contrary to the logical expectation that abstinence before transplantation would improve outcome, this study does not demonstrate a statistically significant difference between the survival of transplant recipients who abstained from alcohol for at least 6 mo before the transplantation and those who did not (Table 2). It is interesting to note, however, that alcohol consumption in the immediate pretransplantation period did predict postoperative recidivism.

This study also clearly demonstrates that the recidivism rate for alcoholism after OLT is rather low. The reason is unclear, but the trauma of a progressive decline in health before transplantation, followed by the drama of facing an operation of the magnitude of OLT may have provided the motivation required for sustained sobriety (10). Adequate social, family and psychiatric support systems have no doubt substantially helped most of these survivors avoid an alcohol abuse relapse.

Liver transplantation results in this series confirm the foresight of the consensus panel and show that alcoholic patients with advanced liver disease can be transplanted successfully with a reasonable expectation of subsequent abstinence, employability and quality of life. These data also show that the 1-yr survival rate for individuals transplanted for alcoholic liver disease (74\%) does not differ from that of persons receiving transplants for other hepatocellular diseases. In fact, the survival rate is better than that reported for those receiving transplants for liver disease caused by chronic hepatitis B infection, HCC or cholangiocarcinoma (11). Most importantly, most of these patients have been successfully rehabilitated and are able to go back to work or work in the home, and their quality of life appears to be equivalent to that of their nonalcoholic counterparts. 
Many chronic alcoholic patients die of end-stage alcoholic liver disease or one of its complications such as bleeding varices or the hepatorenal syndrome (12). These deaths may be preventable now that the therapy of liver transplantation is available for at least some of these patients. It should be noted, too, that most physicians provide therapy to smokers, the obese, drug addicts or persons with venereal disease, and portacaval shunting or sclerotherapy treatment is provided to patients with cirrhosis who do not claim they will lead a life of abstinence (13). Available data suggest that the recidivism rates for smoking, drug addiction and obesity may be equal to, or greater than, those for alcoholism, but these relapse rates are not used to deny medical care to those who require it. Unwillingness to offer liver transplantation to individuals with alcoholic liver disease because of a failure to demonstrate long-term abstinence, particularly in view of the great risk for death without OLT, appears difficult to defend.

\section{REFERENCES}

1. Starzl TE, Iwatsuki S, Van Thiel DH, Gartner JC, Zitelli BJ, Malatack JJ, Schade RR, et al. Evolution of liver transplantation. Hepatology 1982;2:614-636. [PubMed: 6749635]

2. Dindzans VJ, Schade RR, Gavaler JS, Tarter RE, Van Thiel DH. Liver transplantation: a primer for the practicing gastroenterologist: Parts 1 and 2. Dig Dis Sci 1989;34:2-8. 161-166. [PubMed: 2642794]

3. Starzl TE, Iwatsuki S, Shaw BW Jr. Gordon RD, Esquivel C. Liver transplantation in the cyclosporine era. Prog Allerg 1986;38:366-434.

4. Consensus panel of the consensus development conference on liver transplantation, National Institutes of Health consensus development statement. Liver transplantation. June 20-23,1983. Hepatology 1984;49(Suppl):107s-110s.

5. Maddrey, WC.; Friedman, LS.; Munoz, SJ.; Hahn, EG. Selection of the patient and timing of surgery. In: Maddrey, WC., editor. Transplantation of liver. Elsevier Science Publishing Co., Inc.; New York: 1988. p. 40-45.

6. Liver transplantation. Consensus Conference. JAMA 1983;250:2962-2964.

7. Schenker S. Medical treatment vs. transplantation in liver disorders. Hepatology 1984;4:102s-106s. [PubMed: 6363253]

8. Soterakis J, Resnick RH, Iber FL. Effects of alcohol abstinence on survival in cirrhotic portal hypertension. Lancet 1973;2:65-67.

9. Pande NV, Resnick RH, Yee W, Eckardt VP, Shurberg JL. Cirrhotic portal hypertension: morbidity of continued alcoholism. Gastroenterology 1978;74:64-69. [PubMed: 618432]

10. Starzl TE, Van Thiel DH, Tzakis A, Iwatsuki S, Todo S, Marsh JW, Konery B, et al. Orthotopic liver transplantation for alcoholic cirrhosis. JAMA 1988;260:2542-2544. [PubMed: 3050180]

11. Iwatsuki S, Starzl TE, Todo S, Gordon RD, Esquivel CO, Tzakis AG, Makowka L, et al. Experience in 1000 liver transplants under cyeiosporin-steroid therapy: a survival report. Transplant Proc 1988;20:498-505. [PubMed: 3279643]

12. Mendenhall, CL. Alcoholic hepatitis. In: Schiff, L.; Schiff, ER., editors. Diseases of the liver. Vol. 18. JB Lippincott; Philadelphia: 1987. p. 669-686.

13. Atterbury CE. The alcoholic in the lifeboat. Should drinkers be candidates for liver transplantation? J Clin Gastroenterol 1986;8:1-4. [PubMed: 3517127] 


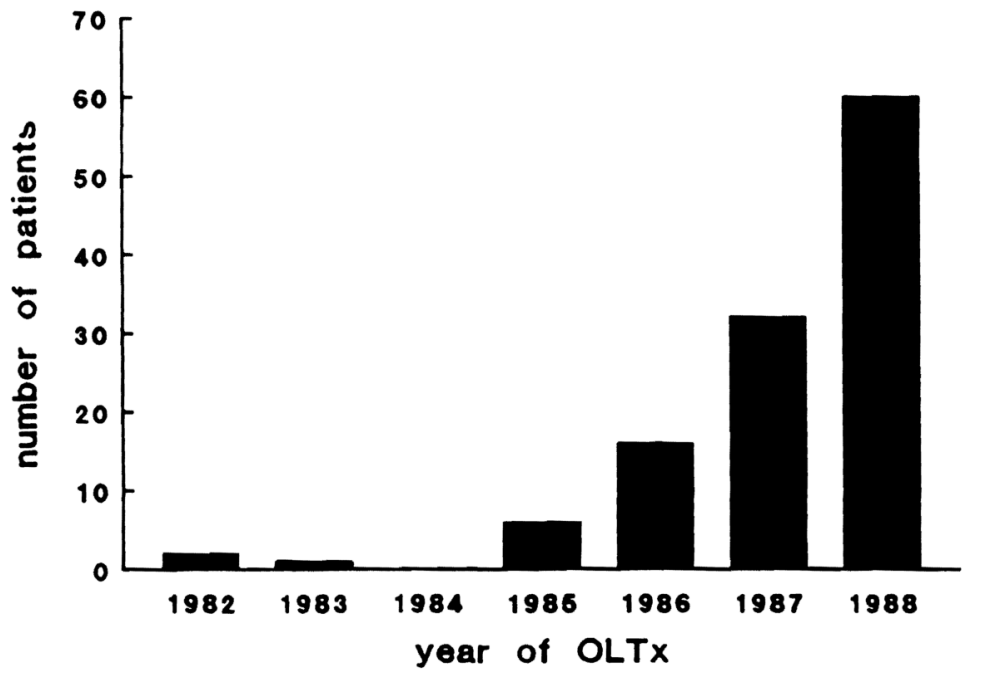

Fig. 1.

Patients transplanted annually for alcoholic liver disease at the University of Pittsburgh. Note that time period shown exceeds the duration of this study. 


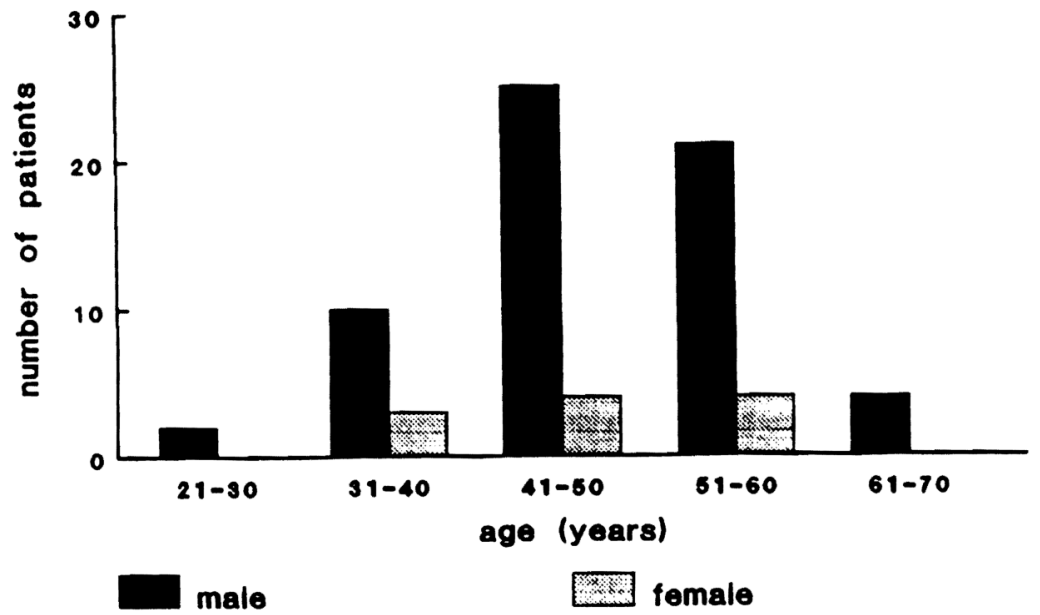

Fig. 2.

Age and sex distribution of persons receiving transplants for alcoholic liver disease at the University of Pittsburgh during period of study. 


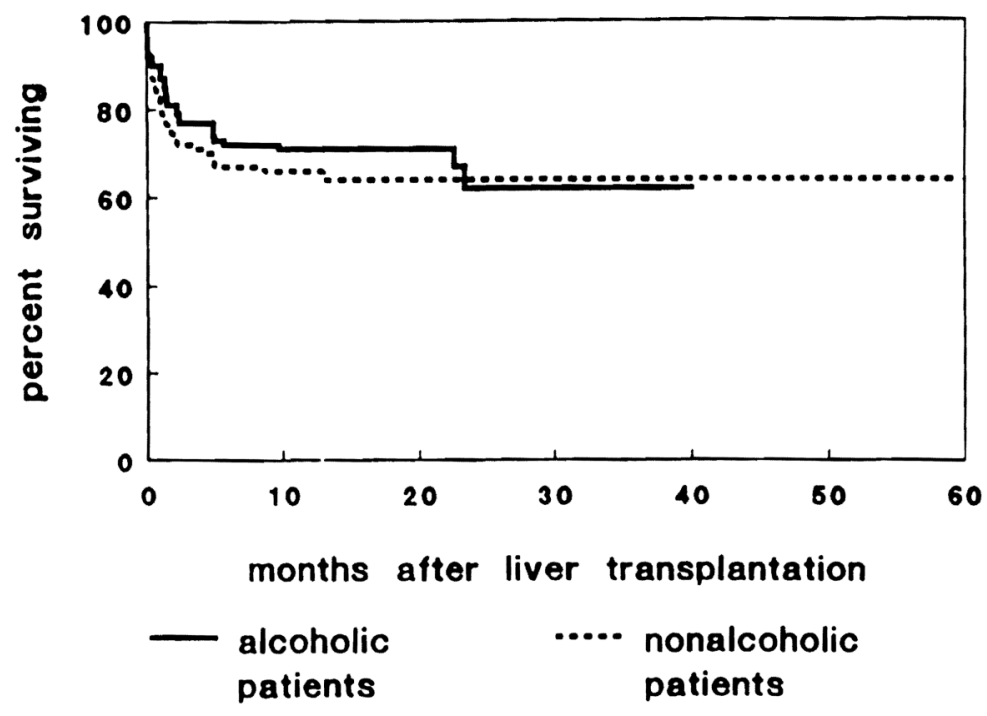

Fig. 3.

Kapian-Meier survival plots for 73 persons transplanted between July 1982 and April 1988 for alcoholic liver disease compared with survival of 73 individuals with nonalcoholic liver disease matched for age, sex and time of transplantation. 
Table 1

Child's-Pugh class and mortality of alcoholic transplant recipients

\begin{tabular}{lrll}
\hline Class & Total & Survived & Deceased \\
\hline A & 2 & 2 & 0 \\
B & 24 & 19 & 5 \\
C & $\underline{47}$ & $\underline{31}$ & $\underline{16}$ \\
TOTAL & 73 & $52(71 \%)$ & $21(29 \%)$ \\
\hline
\end{tabular}




\section{Table 2}

Characteristics of alcoholic patients treated

\begin{tabular}{lrr}
\hline $\begin{array}{l}\text { Duration of alcoholic abstinence } \\
\text { before OLT }\end{array}$ & Survived & Deceased \\
\hline$<6$ mo $(\mathrm{n}=11)$ & 7 & 4 \\
6 to 24 mo $(\mathrm{n}=29)$ & 25 & 4 \\
$>24$ mo $(\mathrm{n}=33)$ & 20 & 13 \\
TOTAL & 52 & 21 \\
\hline
\end{tabular}

\footnotetext{
${ }^{a}$ No significant difference in survival noted among the three groups, whether compared as shown in the table or with groups 6 to 24 mo and $>24$ mo combined into a single group and compared to the $<6$ mo group.
} 
Table 3

Cause of death after OLT for alcoholic liver disease

\begin{tabular}{lccr}
\hline & $\begin{array}{c}\text { Patient dead <3 mo after } \\
\text { transplantation }(\mathbf{n}=\mathbf{1 3})\end{array}$ & $\begin{array}{c}\text { Patient dead }>\mathbf{3} \text { mo after } \\
\text { transplantation }(\mathbf{n}=\mathbf{8})\end{array}$ & Total \\
\hline Intraoperative death & & & \\
$\quad$ Cardiac arrest & 3 & $\mathrm{NA}^{a}$ & 3 \\
$\quad$ Bleeding & $\underline{3}$ & $\mathrm{NA}$ & $\underline{3}$ \\
TOTAL & 6 & & 6 \\
Postoperative death & & & \\
Spnsis & 5 & 4 & 9 \\
Rejection & 0 & 2 & 2 \\
Pulmonary embolism & 0 & 1 & 1 \\
Intracranial hemorrhage & 1 & 1 & 2 \\
$\quad$ Gastrointestinal bleeding & 1 & $\underline{0}$ & 1 \\
TOTAL & 7 & 8 & 15 \\
\hline
\end{tabular}

${ }^{a} \mathrm{NA}=$ not applicable. 
Table 4

Alcohol consumption after liver transplantation

\begin{tabular}{cll}
\hline & Alcoholics & Nonalcoholics \\
\hline Surviving patients & 52 & 49 \\
Not drinking & $46(88.5 \%)$ & $39(79.6 \%)$ \\
Drinking & $6(11.5 \%)$ & $10(20.4 \%)$ \\
Social drinking & 4 & 9 \\
Moderate drinking & 2 & 1 \\
Excessive drinking & 0 & 0 \\
\hline
\end{tabular}


Table 5

Agreement between patients, spouses and physicians on alcohol consumption

\begin{tabular}{lrrr}
\hline & Patients & Spouses & Physicians \\
\hline None & 48 & 48 & 46 \\
Social & 3 & 3 & 4 \\
Moderate & 1 & 1 & 2 \\
Excessive & 0 & 0 & 0 \\
\hline
\end{tabular}




\section{Table 6}

Working status after OLT

\begin{tabular}{lrr}
\hline Working status & Alcoholics & Nonalcoholics \\
\hline Full time & 13 & 14 \\
Part time & 10 & 6 \\
Self-employed & 5 & 2 \\
Housework & 11 & 11 \\
Unable to work & 11 & 14 \\
Willing to work, but unable to find job & $\underline{2}$ & $\underline{2}$ \\
TOTAL & 52 & 49 \\
\hline
\end{tabular}


Table 7

Marital status of liver transplant recipients

\begin{tabular}{lcccc}
\hline & Married & Divorced & Single & Widow/er \\
\hline Alcoholic liver disease $(\mathrm{n}=52)$ & 45 & 3 & 3 & 1 \\
Nonalcoholic liver disease $(\mathrm{n}=49)$ & 31 & 7 & 9 & 2 \\
\hline
\end{tabular}




\section{Table 8}

Serum AST and ALT levels of the surviving individuals at the last follow-up

\begin{tabular}{lrrrrrr}
\hline & $\begin{array}{c}\text { Alcoholics } \\
(\mathbf{n}=\mathbf{5 2})\end{array}$ & & \multicolumn{2}{c}{$\begin{array}{c}\text { Nonalcoholics } \\
(\mathbf{n}=\mathbf{4 9})\end{array}$} \\
\cline { 2 - 3 } \cline { 6 - 7 } & AST & ALT & & AST & ALT \\
\hline Normal & 36 & 36 & 27 & 20 \\
$<2$ times upper limit of normal & 9 & 7 & 12 & 13 \\
$>2$ times upper limit normal & 7 & 9 & 10 & 16 \\
\hline
\end{tabular}

